\title{
The application of flow cytometry to the study of bacterial responses to antibiotics
}

\author{
V. A. GANT, G. WARNES*, I. PHILLIPS and G. F. SAVIDGE* \\ Division of Microbiology, UMDS (St Thomas's Campus) and * Department of Coagulation and Human Cell Biology, \\ St Thomas' Hospital, Lambeth Palace Road, London SE1 $7 E H$
}

\begin{abstract}
Summary. Experiments were performed to determine whether a modern flow cytometer could be used to study bacterial populations in suspension, with particular reference to their morphological characteristics and their responses to antibiotics. The FACScan, a commercial benchtop flow cytometer fitted with an air-cooled laser, designed primarily for the study of eukaryotic peripheral blood mononuclear cells, yielded reproducible data relating to bacterial shape and internal architecture. It was sensitive enough to detect changes in bacterial morphology on entry into the growth cycle and after exposure to antibiotics. Antibioticinduced morphological changes affecting subpopulations of bacteria were sufficiently specific to allow differentiation between antibiotics with different cell-wall enzyme targets. Simultaneously, the effect of such antibiotics on the integrity of the outer cell membrane of Escherichia coli was assessed by measurement of the association of the nucleic acid-binding dye propidium iodide with the bacteria. These experiments demonstrated complex patterns of probable cell-wall leakage, related to the modes of action of the antibiotics. The FACScan is a useful and sensitive tool for the study of the morphology and physiology of bacterial populations in suspension, and is especially applicable to the study of antibiotic action.
\end{abstract}

\section{Introduction}

Experimental methods developed over the last century to observe and characterise growing microorganisms have largely concerned themselves with the behaviour of bacterial populations. Thus, whilst methods such as turbidimetry and biochemical analysis of broth cultures yield valuable information on the average behaviour of bacterial populations, such techniques cannot yield information specific to individual cells, which are often heterogeneous in terms of age, growth rate and metabolism. Such heterogeneity becomes important in studies of mechanisms of antibiotic action in which, for example, antimicrobial susceptibility may be profoundly influenced by growth rate. ${ }^{1}$ Whereas this problem can be addressed by synchronisation of the bacterial population in a continuous chemostat culture, ${ }^{2}$ these conditions are unlikely to prevail in vivo. Thus, caution is necessary in assessing conclusions drawn from chemostat-synchronised cultures, on the grounds of "artificiality", or from "outbred" cultures, on the grounds that the results are an "average" for the whole population.

This situation has arisen because of the technical difficulties of studying individual microbial cells. Such practical limitations were also true for eukaryotic cells before the advent of techniques including micromanipulation, in-vitro cell monolayer perfusion, intracellular probing of subcellular organelle biochemical pathways, and flow cytometry, each of which can be applied to individual eukaryotic cells. We have investigated the suitability of flow cytometry for the study of bacteria to determine whether this technique might be applicable to the study of the morphology and physiology of individual cells. We sought to determine whether a commercially available and unaltered benchtop flow cytometer could: (a) detect bacterial cells with a sufficiently low signal to noise ratio; (b) detect changes in shape induced by antibiotics active against cell-wall components; and (c) detect antibiotic-induced changes in bacterial membrane permeability.

\section{Materials and methods}

\section{Bacteria and culture conditions}

Escherichia coli clinical strain no. 33110 , isolated from urine and blood from a patient at St Thomas' Hospital, was used throughout this study. The MICs for this organism, determined by the microdilution method in which the end-point is represented by visible growth from an inoculum of $c .10^{4} \mathrm{cfu}$ in IsoSensitest

Received 26 Nov. 1992; accepted 13 Jan. 1993. 
Broth (Oxoid; CM471B) after overnight incubation at $37^{\circ} \mathrm{C}$ in air, were $(\mathrm{mg} / \mathrm{L})$ : gentamicin 1 , ampicillin 4 , cefotaxime 0.03 mecillinam 0.8 and ciprofloxacin 0.016 . The strain was subcultured from nutrient agar slopes and grown for $1 \mathrm{~h}$ at $37^{\circ} \mathrm{C}$ in Nutrient Broth (no. 0510B; Southern Group Laboratories, Corby, Northamptonshire) which had been filtered through sterile $0 \cdot 22-\mu \mathrm{m}$ filters (Millipore) to produce a starting culture with a turbidity of $c .0 .5$ Macfarland units.

\section{Estimation of bacterial morphology}

The starting culture was diluted 1 in 10 in fresh filtered nutrient broth and either analysed immediately or incubated for a further 1 or $3 \mathrm{~h} ; 20-\mu \mathrm{l}$ samples were transferred to Falcon 2054 round-bottomed polystyrene tubes designed to fit the cytometer input nozzle, referred to hereinafter as FACS tubes (Becton Dickinson, Cowley, Oxfordshire), containing $1 \mathrm{ml}$ of a filtered high purity sterile isotonic solution buffered to pH 7.4 (FACSflow, Becton Dickinson), gently inverted, and analysed immediately by flow cytometry. For analysis of the starting culture, the FACS tubes also contained latex beads (Sigma) measuring $3.2 \mu \mathrm{m}$ and $11.9 \mu \mathrm{m}$ to enable the signals from the bacteria to be localised in the graphical representation of the scan and the instrument settings to be optimised.

\section{Measurement of antibiotic effect on the shape and membrane permeability of E. coli cells}

Starting cultures were diluted 1 in 10 in filtered IsoSensitest broth, and $100-\mu \mathrm{l}$ volumes were added to the wells of a 96-well microtitration plate (Costar) into which had been dispensed $100-\mu \mathrm{l}$ volumes of $0.22-\mu \mathrm{m}$ filtered IsoSensitest broth containing doubling dilutions of antibiotics which had been freshly prepared from powders and adjusted for specific activity. Antibiotics tested were: gentamicin, mecillinam, cefotaxime, ampicillin and ciprofloxacin (all supplied by their manufacturers). After incubation for $3 \mathrm{~h}$ at $37^{\circ} \mathrm{C}$ and gentle resuspension, $20-\mu \mathrm{l}$ samples were transferred to FACS tubes containing $0.5 \mathrm{ml}$ of FACSflow buffer and analysed immediately. After incubation for a further $2 \mathrm{~h} 45 \mathrm{~min}, 50 \mu \mathrm{l}$ of a solution of propidium iodide (Molecular probes; Eugene, OR, USA) $250 \mathrm{mg} /$ $\mathrm{L}$ in Hanks's Balanced Salts Solution were added to the duplicate wells. These cultures were re-incubated for $15 \mathrm{~min}$ and analysed as described above, with further dilution as necessary in FACSflow to produce a cytometer event rate of not more than $500 / \mathrm{s}$.

\section{FACS analysis}

Forward and side light scatter and fluorescence data were acquired with a physically unmodified FACScan flow cytometer (Becton Dickinson, Mountain View, CA, USA), fitted with a $15 \mathrm{~mW}$ air-cooled argon laser, with the standard fluidics orifice and the following

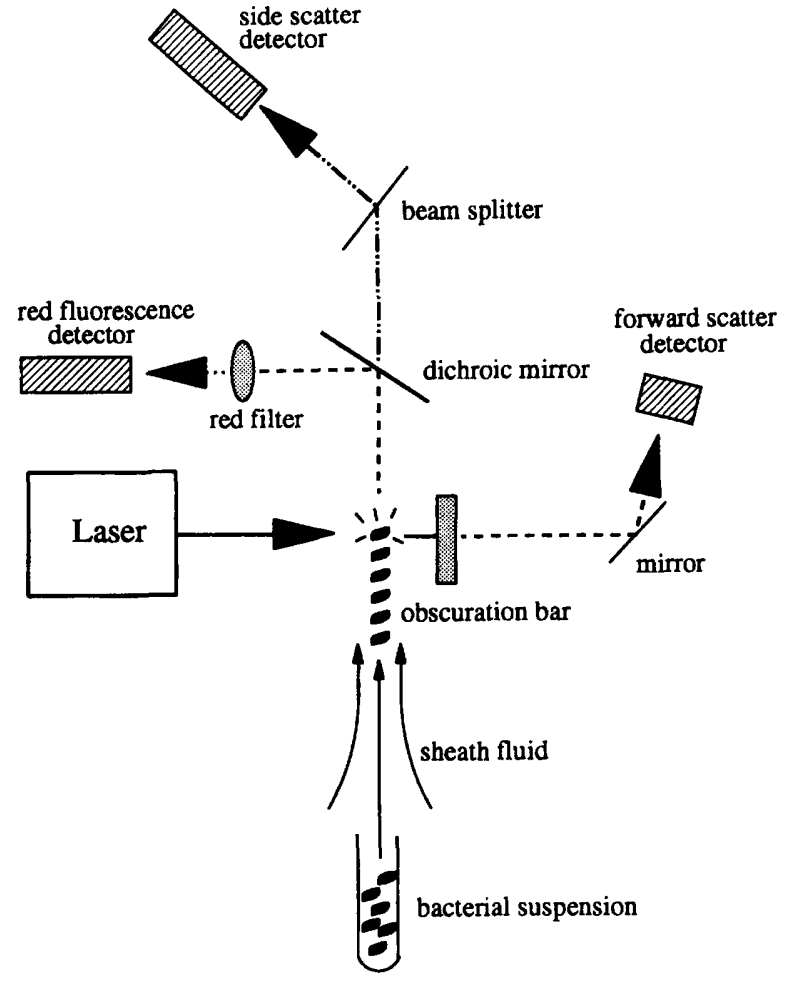

Fig. 1. Schematic simplified representation of the optical arrangement of the FACScan flow cytometer, showing those elements and detectors relevant to this study.

optical arrangement: after a short band-pass $560-\mathrm{nm}$ dichroic mirror, the beam is split and reaches four photomultiplier tubes; in this study, information was collected from the photodiode, placed directly in front of the laser beam (forward scatter), a photomultiplier tube detecting light scattered at $90^{\circ}$ to the laser beam (side scatter) and a second photomultiplier tube detecting far red fluorescence via a 650 -nm filter. All detector amplifiers were set on logarithmic gain mode; the forward scatter amplifier was set to the E00 amplification mode. A minimum gate on forward scatter criteria was set to exclude subcellular debris and optical "noise". Any particle generating a forward scatter photomultiplier pulse exceeding the minimum gate amplitude was subsequently defined as an event, and recorded on to magneto-optical disk together with data collected simultaneously relating to the amplitudes of the pulses generated by the other photomultiplier tubes; at least 10000 events were recorded and saved on magneto-optical disk. Subsequent data analysis was performed with Lysis II software (Becton Dickinson). The data shown here represent two- and three-dimensional contour plots of 10000 events. Fig. 1 shows a schematic diagram of the laser and detector arrangement.

\section{Results}

Flow cytometric profiles of bacteria

Fig. 2 shows two-dimensional representations of the 


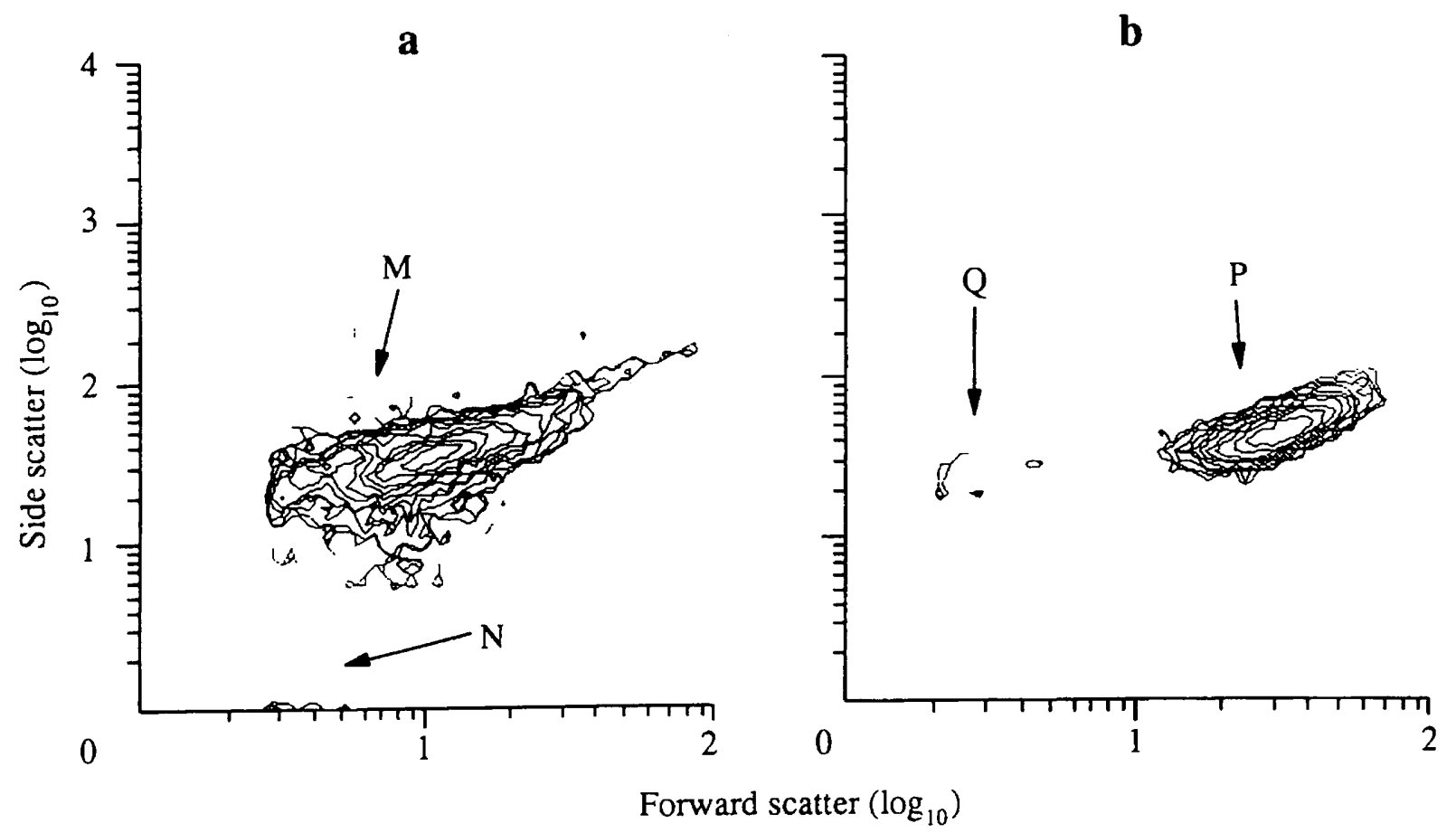

Fig. 2. Two-dimensional density contour of $E$. coli after incubation for (a) 1 and (b) $3 \mathrm{~h} ; 10000$ bacteria were analysed. $\mathrm{M}$, normal bacterial cells; $N$, subcellular particles; $P$, population shifted in forward scatter; $Q$, subcellular particles.

light scattering properties of a suspension culture of $10000 \mathrm{E}$. coli cells after incubation for 1 and $3 \mathrm{~h}$. The density contours represent the number and position of the bacteria in a plot of forward scatter ( $x$ axis) versus side scatter ( $y$ axis). Sterile filtered nutrient broth analysed in the same fashion for the same period ( $c$. $20 \mathrm{~s})$ yielded no more than 20 events. Fig. 2a shows that the flow cytometer can distinguish the bacterial population (population $\mathrm{M}$ ) from optical and electronic "noise". However, such "noise" is present at forward scatter values $<10$. Therefore, such signals were gated out electronically at the acquisition stage, and are not displayed. Minimal contamination from subcellular particles can be seen along the origin of the $y$-axis in the 1-h sample (population $\mathrm{N}$ ), and is far removed from the events representing whole bacteria. By $3 \mathrm{~h}$, a shift in the population of bacteria in both forward and side scatter characteristics can be seen; this mostly represents a shift in forward scatter characteristics (population P; fig. 2b). A small number of events was recorded close to the axis of origin, which may also represent subcellular particles (population Q). The shift in forward and side scatter observed after incubation for a further $2 \mathrm{~h}$ may represent the bacterial population moving into the log phase of growth; individual events may represent larger individual organisms, some of which may be undergoing septation and division. Fig. $2 \mathrm{~b}$ shows again that the flow cytometer can detect these bacteria and distinguish them from optical and electronic "noise".

Fig. 3 shows data obtained from a starting culture to which latex beads had been added, displayed as a three-dimensional contour plot in which the axes represent, on a logarithmic scale, forward and side

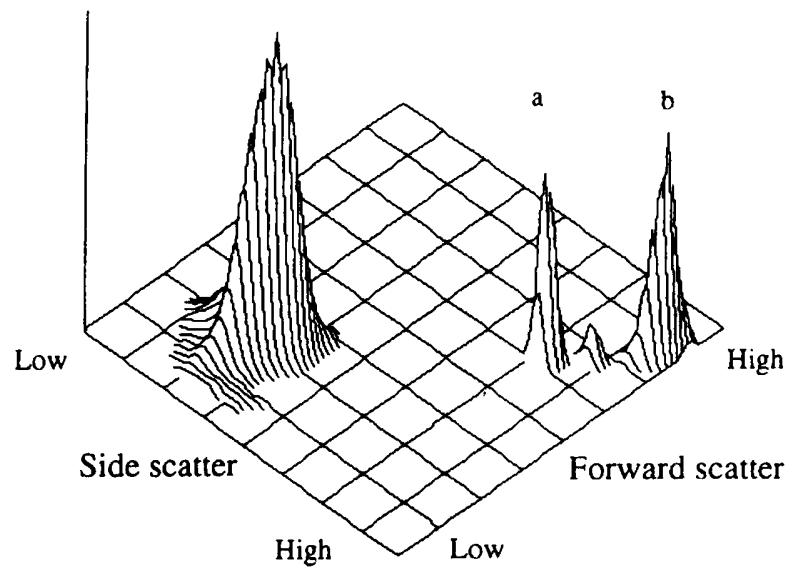

Fig. 3. Three-dimensional contour graph of a starting culture of $E$. coli with latex beads of (a) $3.2 \mu \mathrm{m}$ and (b) $11.9 \mu \mathrm{m}$.

scatter measurements of the particles in suspension, and the height of the contours represents the number of events assigned to a particular forward-side scatter value. It shows a tight population of bacteria, easily separated from the latex beads, which have much larger forward-side scatter characteristics. The small peak between the two bead populations represents doublets of $3 \cdot 2-\mu \mathrm{m}$ beads.

Effect of antibiotics on the light scattering properties of E. coli

Fig. 4 shows the effects of antibiotics on the light 
$3 \mathbf{h}$

a

b

c

d

e

f

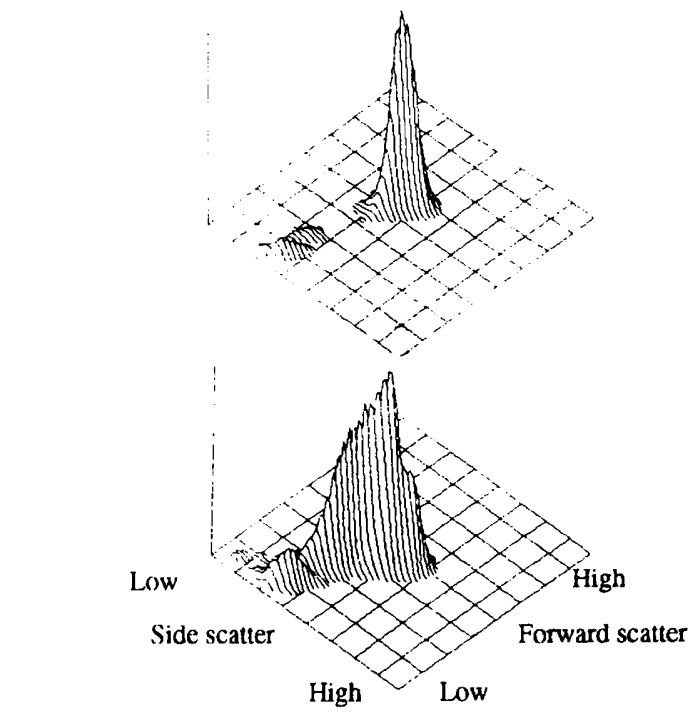

$6 \mathrm{~h}$
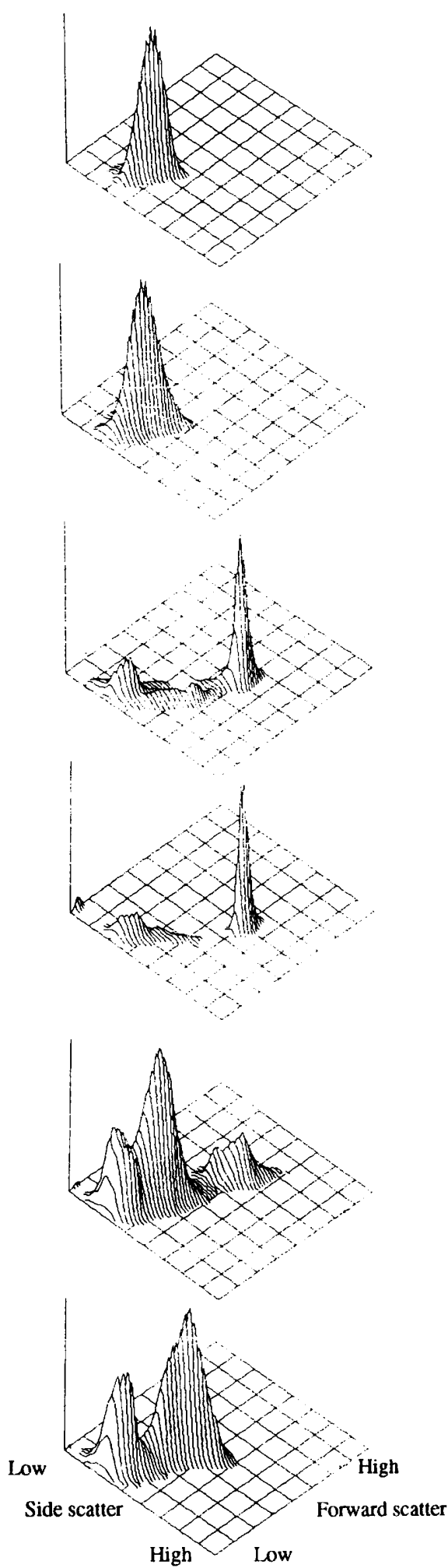

Fig. 4. Three-dimensional contour graphs of $E$. coli after incubation for 3 and $6 \mathbf{h}: \mathbf{a}$, control; b, gentamicin; $\mathbf{c}$, ampicillin; d, cefotaxime; mecillinam; $\mathbf{f}$; ciprofloxacin. Each antibiotic was used at its MIC for this strain.

scattering properties of $E$. coli cultures incubated for 3 and $6 \mathrm{~h}$ with antibiotics; for this representative experiment, instrument and software settings were identical to those used for the experiment shown in fig. 3. Each antibiotic was present at its MIC for the test strain. At 3 h, control cultures showed a tightly defined population, with a few events falling within the area of the graph associated with subcellular particles. After a further $3 \mathrm{~h}$, no change in the co-ordinates of the population was seen. The subsequent contour plots in 


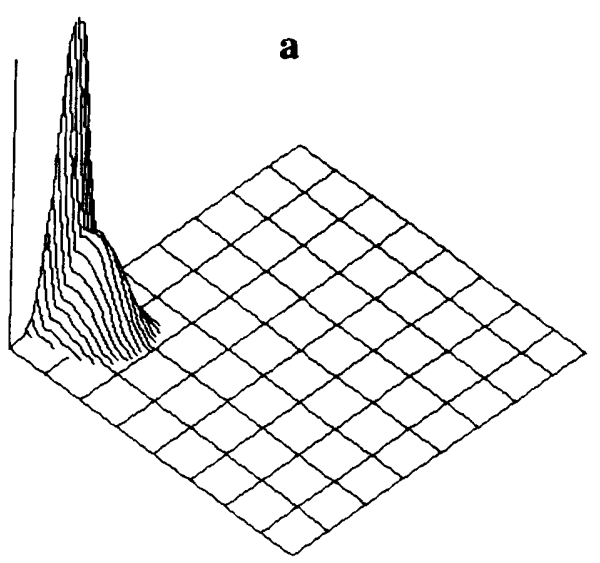

c

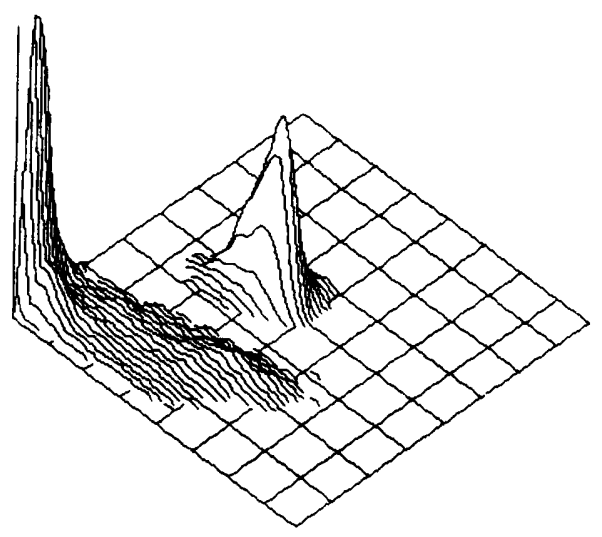

e

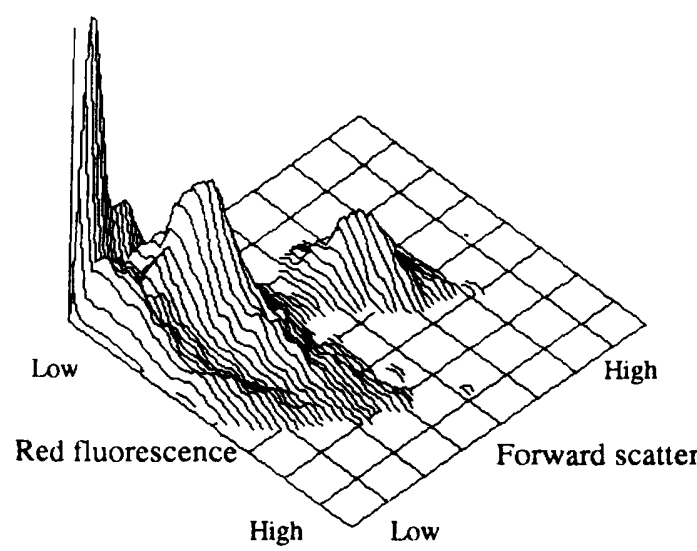

b

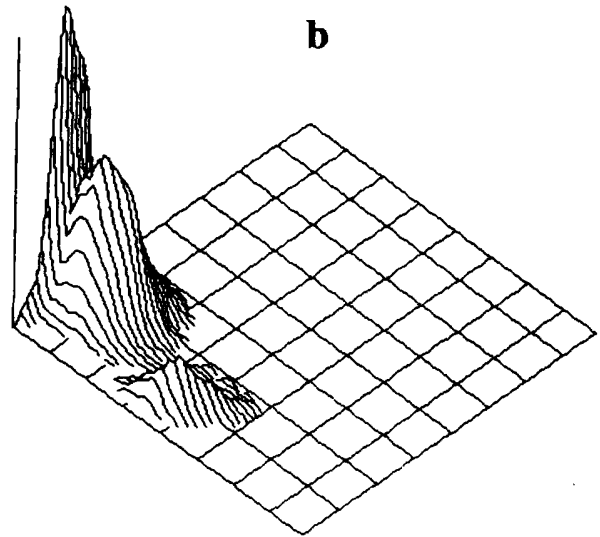

d

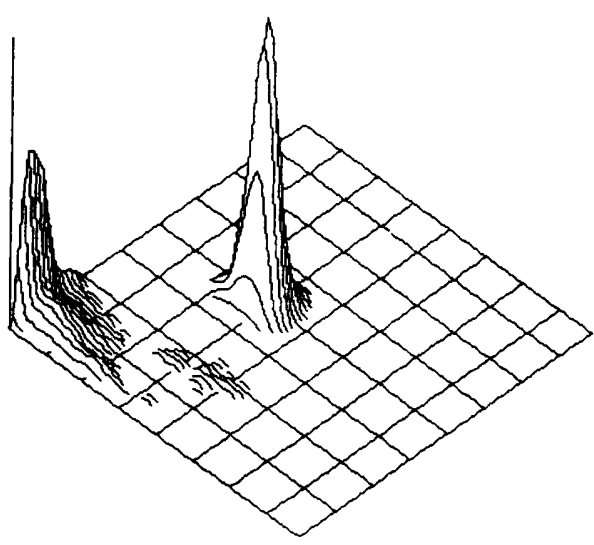

f

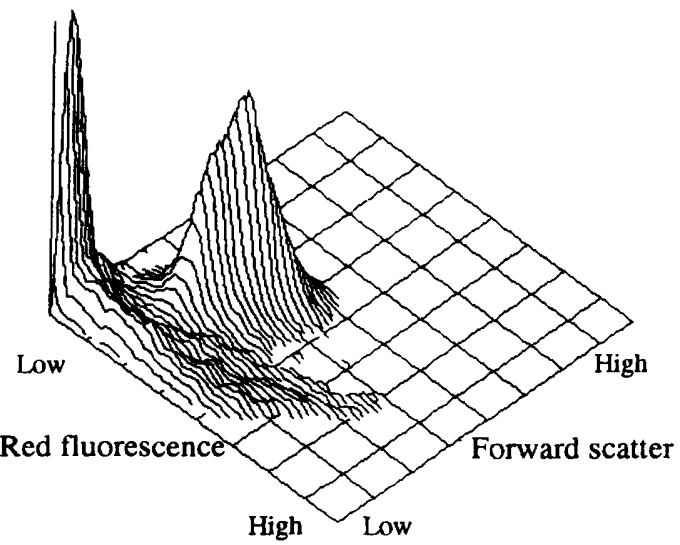

Fig. 5. Three-dimensional contour graphs of $E$. coli after incubation for $6 \mathrm{~h}$, showing forward scatter plotted against red fiuorescence (reflecting binding of propidium iodide by nucleic acid): a, control; b, gentamicin; $\mathbf{c}$, ampicillin; d, cefotaxime; e, mecillinam; f, ciprofloxacin.

fig. 4 represent results obtained with bacteria exposed to antibiotics; many demonstrate profound changes in the forward-side scatter co-ordinates of the bacterial populations, which fall into three broad groups that reflect the modes of action of the antibiotics. For all the profiles obtained in the presence of antibiotics, longer acquisition times were necessary (c. $30 \mathrm{~s}$, compared with $3 \mathrm{~s}$ for the control population) because of the smaller concentration of bacteria present; the profiles represent the characteristics of the remaining bacterial populations.

When compared with the control culture, gentamicin induced little change in the profile of the remaining bacterial population (fig. $4 \mathrm{~b}$ ). However, a larger component of the culture occupied a low forward-side scatter zone, associated with subcellular particles. The monitor display at the time of acquisition also showed that a large component of 
detected events fell below the electronic gate set for the lower limit of forward scatter; these events, representing particles measuring $<0.1 \mu \mathrm{m}$, were not retained for analysis and are not displayed here. Similarly, by $6 \mathrm{~h}$, a population of events approximating to the control population's co-ordinates persisted.

By contrast, the three antibiotics active against bacterial cell walls (ampicillin, cefotaxime and mecillinam) induced profound changes in the majority of the population by $3 \mathrm{~h}$ (figs. $4 \mathrm{c}$, d and e). With ampicillin, the majority of the culture was equally shifted in both forward and side scatter characteristics, with no cells remaining in the part of the plot occupied by the control population. By $6 \mathrm{~h}$, a tail of events with predominantly shifted side scatter characteristics had appeared; this tail fell back to a second smaller population representing subcellular bacterial particles. Similarly, cefotaxime induced a shift in both scatter parameters at $3 \mathrm{~h}$, with no events remaining in the area of the display associated with control bacteria. By $6 \mathrm{~h}$ only a small population of shifted events remained; a new population of events appeared in a region associated with subcellular fragments.

With mecillinam, after $3 \mathrm{~h}$, a well defined population of events with much higher forward scatter and moderately high side scatter had appeared; only a small number of events remained in the area of coordinates occupied by the control population. By $6 \mathrm{~h}$, this well defined population had mostly given way to a new population of events with smaller scatter coordinates, contiguous with a population of subcellular particles. Analysis after further incubation revealed a "falling back" of large events into this area, with the eventual disappearance of events large enough to represent bacteria (data not shown).

Fig. 4f shows the effect of the presence of ciprofloxacin at 3 and $6 \mathrm{~h}$. There was a homogeneous shift in scatter characteristics unlike that seen with antibiotics active against cell-wall components, with the appearance of a small number of events in the subcellular region of the plot and a large continuous shift in the light scattering properties, and a "hump" of events at the upper end of the plot. A proportion of this shifted component remained at $6 \mathrm{~h}$, but a large number of events had now appeared in the subcellular region of the plot.

\section{Effect of antibiotics on the permeability of the E. coli cell wall}

Fig. 5 shows representative three-dimensional contour plots relating the ability of these bacteria to exclude the nucleic acid-binding dye propidium iodide to their forward light scatter characteristics after incubation in the presence and absence of antibiotic for $6 \mathrm{~h}$. Unlike the control cultures which took up very little dye, all antibiotics tested resulted in significant numbers of events that exhibited red fluorescence, indicating increased access of the dye to bacterial DNA or RNA.
Bacteria incubated in the presence of gentamicin produced a more complex profile of peaks, indicating at least two separate populations. The majority of events fell within the area associated with "healthy" cells, i.e., normal forward scatter characteristics and no, or minimal, red fluorescence. However, unlike the control population, a small proportion of this population tailed off into a region of moderately increased red fluorescence. A second population of events displayed high red fluorescence, and was associated with scatter characteristics suggesting the presence of subcellular particles.

Exposure to ampicillin resulted in the appearance of the well-defined population, shown in fig. $5 \mathrm{c}$, which displayed strong red fluorescence. This antibiotic also induced a continuum of particles with intense fluorescence with forward scatter properties suggesting single organisms, tailing off into a region of the plot corresponding to subcellular particles; a major peak of non-fluorescent debris, presumably containing no nucleic acids, was also seen. A similar profile was obtained with cefotaxime, although in this case no bacteria with "normal" light scatter and fluorescence characteristics were evident (fig. 5d). In contrast, exposure to mecillinam resulted in a peak of large events which had taken up the fluorescent dye, with a second more prominent peak of bacteria with normal forward scatter characteristics displaying a continuum of increased fluorescence, and a large contribution of events with the characteristics of subcellular particles, many of which contained nucleic acid (fig. 5e).

The majority of whole bacteria exposed to ciprofloxacin also displayed abnormal red fluorescence, together with a smaller component of cells with "normal" forward scatter characteristics displaying a more scattered continuum of red fluorescence, and a peak of non-fluorescing subcellular particles (fig. $5 f$ ).

\section{Discussion}

Despite the advent of flow cytometers theoretically sensitive enough to detect bacteria and analyse their morphology, little work on this subject has emerged. This is surprising in view of the need in microbiology for a technique that will yield information on the often complex mixtures of cells present in natural environments in vivo, and in bacterial cultures. This is even more relevant in studies of the effects of antibacterial agents, in which it is important to obtain information on the various subpopulations present within a bacterial culture, some of which may behave quite differently. Furthermore, as incubation progresses and nutrient limitation becomes important, bacterial envelopes change in structure, and sensitivity to antibacterial agents is reduced. ${ }^{1-3}$ Flow cytometry would seem an ideal tool to investigate such processes, but only a few groups of workers have reported its usefulness, probably because it is still considered technically difficult. Phillips and Martin ${ }^{4}$ commented 
on the physical limits imposed by a commercial flow cytometer for the detection of bacteria in suspension -although these workers were attempting to identify small numbers of undefined bacteria in large volumes of fluid, rather than investigating the properties of pure cultures. Other workers have used purposedesigned cytometers to detect low numbers of $E$. coli in peripheral blood, ${ }^{5}$ Listeria monocytogenes in milk, ${ }^{6}$ Legionella spp., ${ }^{7}$ and assorted aquatic bacteria in environmental waters. ${ }^{8}$ Cohen and Sahar ${ }^{9}$ have also described a flow cytometric method for the detection of bacteria in body fluids and exudates. In each of these studies, the technique has been used as a tool for detecting and differentiating bacteria from other particles, rather than as a probe of bacterial form and function. However, Steen et $a l .{ }^{10}$ analysed the bacterial cell cycle with special reference to bacterial ploidy and the influence of rifampicin with DNA-specific dyes, but again with a purpose-built machine designed to achieve the low signal-to-noise ratios necessary for such work. Such a dedicated machine is now available commercially (Skatron Argus, Tranby, Norway).

We have demonstrated that the FACScan, a benchtop flow cytometer with many established applications in eukaryotic cell biology, can detect viable, unstained bacteria and differentiate them from optical and electronic "noise". The results shown here were obtained with instrument settings similar to those used for platelet analysis, except that logarithmic amplification of photomultiplier outputs was used because no useful information could be obtained with linear amplification settings, as the co-efficient of variation in the light scattering properties of bacteria was too large for the entire bacterial population to be displayed simultaneously. Also notable was the increased forward scatter when broth cultures were incubated from 1 to $3 \mathrm{~h}$. Although no formal experiments were performed to determine the nature of the shift in forward scatter, it is reasonable to assume that figs. 2a and $\mathrm{b}$ represent a population of $E$. coli in lag and growth phase, respectively, a conclusion also reached by Steen with a purpose-built machine. ${ }^{11}$ At present we do not know what the flow cytometer forward and side scatter photomultiplier tube events represent. It would be simplistic to assume that forward scatter is simply proportional to size, which may be true for spherical particles. There is an approximately linear relationship between light scattering properties and total protein content of individual bacteria. ${ }^{12}$ Similar uncertainty concerns side scatter amplitude, which may bear a relationship to the "internal complexity" of eukaryotic cells, in which well-defined subcellular organelles are "seen" through a lipid bilayer of different composition to that of bacteria. However, bacterial cell walls may be "opaque" to light, and side scatter may reflect the optical density of the cell wall. The FACScan detects only the amplitude of each individual photomultiplier pulse; for forward scatter, this may differ with the angle at which a bacillus transects the laser beam (end or side) - a concept that does not apply to more fluid and spherical eukaryotic cells. In this context, we have found that gram-positive cocci differ from gram-negative bacilli in their light scattering properties (unpublished observations). Despite these reservations, the data show that the FACScan can readily detect bacteria, and distinguish them from optical and electronic noise. The low signalto-noise ratio can be achieved only with the use of filtered broths. Further investigation is needed to define the elements of bacterial morphology, such as bacterial filamentation in the presence of antibiotics, that determine the profiles obtained.

The antibiotics used in these experiments were chosen to reflect different mechanisms of action: gentamicin is active at the ribosome and indirectly at the cell membrane as a result of incorporation of altered proteins; ciprofloxacin inhibits DNA gyrase; and $\beta$-lactams affect the cell wall (the choice of $\beta$ lactams reflected their different affinity for penicillinbinding proteins). Specific cytometer profiles were obtained for each mode of action. However, the proportion of the original population undergoing such profile changes before lysis is unknown because the concentration of bacteria remaining at the time of analysis was not determined. Furthermore, changes in light scattering were detected when antibiotics were present at $<0.1$ MIC (data not shown), results in agreement with the electronmicroscopic findings of Lorian. ${ }^{12}$ Our results indicate that flow cytometry may have much to offer in this field, and suggest that the technique could be used to assess the mode of action of novel $\beta$-lactam agents. It is easy to use and has the statistical power inherent in analyses of large numbers of bacteria.

The profiles demonstrated considerable heterogeneity in the response of bacterial populations to antibiotics, which may reflect the use of a desynchronised culture, whose individual members displayed varying sensitivity. E. coli cells not exposed to antibiotics excluded the highly polar propidium iodide, which is excluded from eukaryotic cells by the lipid bilayer; this finding is consistent with the barrier function of the bacterial outer membrane. Although fig. 5 demonstrates large differences in the stainability of individual bacteria exposed to antibiotics, it does not indicate what structures are stained. The large peak of events fluorescing with propidium iodide after exposure to gentamicin might indicate defective outer-membrane repair and consequent "leakiness"; the increased fluorescence seen with other antibiotics that affect cell walls may reflect detection of polykaryons that contain more DNA and RNA than a single bacterium. A 100$\mu \mathrm{m}$ filament of $E$. coli may contain $\geqslant 20$ genome copies; it may be no more "leaky" than a single organism, but has more nucleic acid to bind propidium iodide. Martinez et $a l .{ }^{13}$ used flow cytometry to investigate the action of $\beta$-lactams by measuring the shift in ploidy of single bacterial events and found similar variation in genome copy. This requires further study. The ciprofloxacin-induced profile changes in- 
dicate that bacteria exposed to this agent are still capable of morphological change even after $6 \mathrm{~h}$, which is surprising in view of its reportedly rapid lethal effect. Flow cytometric studies of eukaryotic cells have been

\section{References}

1. Tuomanen E, Schwartz J. Penicillin-binding protein 7 and its relationship to lysis of nongrowing Escherichia coli. Bacteriol 1987; 169: 4912-4915.

2. Brown MRW, Collier PJ, Gilbert P. Influence of growth rate on susceptibility to antimicrobial agents: modification of the cell envelope and batch and continuous culture studies. Antimicrob Agents Chemother 1990; 34: 1623-1628.

3. Tuomanen E, Cozens $\mathrm{R}$, Tosch W, Zak O, Tomasz A. The rate of killing of Escherichia coli by $\beta$-lactam antibiotics is strictly proportional to the rate of growth. J Gen Microbiol 1986; 132 : 1297-1304.

4. Phillips AP, Martin KL. Limitations of flow cytometry for the specific detection of bacteria in mixed populations. $J$ Immunol Methods 1988; 106: 109-117.

5. Mansour JD, Robson JA, Arndt CW, Schulte TH. Detection of Escherichia coli in blood using flow cytometry. Cytometry $1985 ; 6$ : 186-190.

6. Donnelly CW, Baigent GJ. Method for flow cytometric detection of Listeria monocytogenes in milk. Appl Environ Microbiol 1986; 52: 689-695. fruitful; our results indicate that such studies applied to prokaryotic cells may be equally rewarding.

We thank Dr E. Power and Miss Anna King for stimulating discussion, and Miss Linda Bethune for MIC determinations.

7. Tyndall RL, Hand RE, Mann RC, Evans C, Jernigan R. Application of flow cytometry to detection and characterisation of Legionella spp. Appl Environ Microbiol 1985; 49: 852-857.

8. Monfort P, Baleux B. Comparison of flow cytometry and epifluorescence microscopy for counting bacteria in aquatic ecosystems. Cytometry 1992; 13: 188-192.

9. Cohen CY, Sahar E. Rapid flow cytometric bacterial detection and determination of susceptibility to amikacin in body fluids and exudates. J Clin Microbiol 1989; 27: 1250-1256.

10. Steen HB, Skarstak, Boye E. Flow cytometry of bacteria: cell cycle kinetics and effects of antibiotics. Ann NY Acad Sci 1983; 468: 329-338.

11. Steen HB. Flow cytometric studies of microorganisms. In: Melamed, Lindmo, Mendelsohn (eds) Flow cytometry and sorting. New York, Wiley-Liss Inc. 1991: 605.

12. Lorian V, Amaral A. Effects of low concentrations of antibiotics. Antibiot Newslett 1990; 7: 65-70.

13. Martinez OV, Gratzner HG, Malinin TI, Ingram M. The effect of some $\beta$-lactam antibiotics on Escherichia coli studied by flow cytometry. Cytometry 1982; 3: 129-133. 